Non-natural Amino Acids

\title{
A Highly Active DNA Polymerase with a Fluorous Core**
}

\author{
Bastian Holzberger, Marina Rubini, Heiko M. Möller, and Andreas Marx*
}

DNA polymerases catalyze all DNA synthesis in the cell ${ }^{[1]}$ and are key tools in important molecular biological core technologies. ${ }^{[2]}$ Apart from naturally available DNA poly merases, several modified DNA polymerases with new characteristics have been developed. To date, directed evolution using the 20 natural amino acids is a promising method for the creation of nucleic acid polymerases with modified properties. ${ }^{[3]}$ Yet, the incorporation of non natural amino acids may lead to enhanced chemical and biological diversity of protein structures and properties by introduction of functional groups that are not represented by the natural amino acids. ${ }^{[4]}$

The use of auxotrophic strains, which lack the ability to biosynthesize one specific natural amino acid, offers the possibility to replace one amino acid by a non natural analogue. After depletion of the natural amino acid in a defined medium, inducing the expression of the target protein with concomitant addition of the non natural analogue leads to the incorporation of the non natural amino acid by selective pressure incorporation. ${ }^{[5]}$

Fluorinated protein synthesis has been extensively stud ied in the past. ${ }^{[6]}$ It has also been shown that trifluorinated amino acid analogues such as trifluoromethionine (TFM) with drastically enhanced hydrophobicity ${ }^{[7]}$ may lead to proteins with novel characteristics. ${ }^{[4]}$ However, efforts to engineer new enzyme functions and properties by use of TFM instead of methionine are hampered, as the replacement of natural amino acids by trifluorinated analogues can lead to unfavorable interactions. Thus, the global substitution of natural amino acids by trifluorinated analogues may cause the naturally evolved protein scaffold to lose correct folding, sufficient stability, or enzymatic activity. ${ }^{[4 a]}$ Hence, only a few examples of proteins are known in which methionine was globally replaced by TFM, ${ }^{[8]}$ including one single example of an enzyme (phage lysozyme) that is $18 \mathrm{kDa}$ in size, in which about $70 \%$ of the three methionine residues are replaced by TFM. $^{[8 \mathrm{~b}]}$

Herein, we present the generation of a multifluorinated DNA polymerase. The $\mathrm{N}$ terminally truncated version of

[*] B. Holzberger, Dr. M. Rubini, Prof. Dr. H. M. Möller, Prof. Dr. A. Marx Department of Chemistry and Konstanz Research School Chemical Biology, University of Konstanz

Universitätsstrasse 10, 78457 Konstanz (Germany)

Fax: (+49) 7531885140

E mail: andreas.marx@uni konstanz.de

Homepage: www.chemie.uni konstanz.de/ agmarx/

[**] We gratefully acknowledge Dr. A. Marquardt (Proteomics Facility, University of Konstanz) for the support with the MS analysis and financial support by the Konstanz Research School Chemical Biology and the DFG.

() Supporting information for this article is available on the WWWW under http://dx.doi.org/10.1002/anie.200905978.
DNA polymerase I from Thermus aquaticus (KlenTaq) is a thermophilic DNA polymerase composed of 540 amino acids $(63 \mathrm{kDa})$, including 13 methionine (Met) residues ${ }^{[9]}$ that were globally replaced by TFM with a substitution level of approximately $82 \%$ (Scheme 1). The multifluorinated Klen
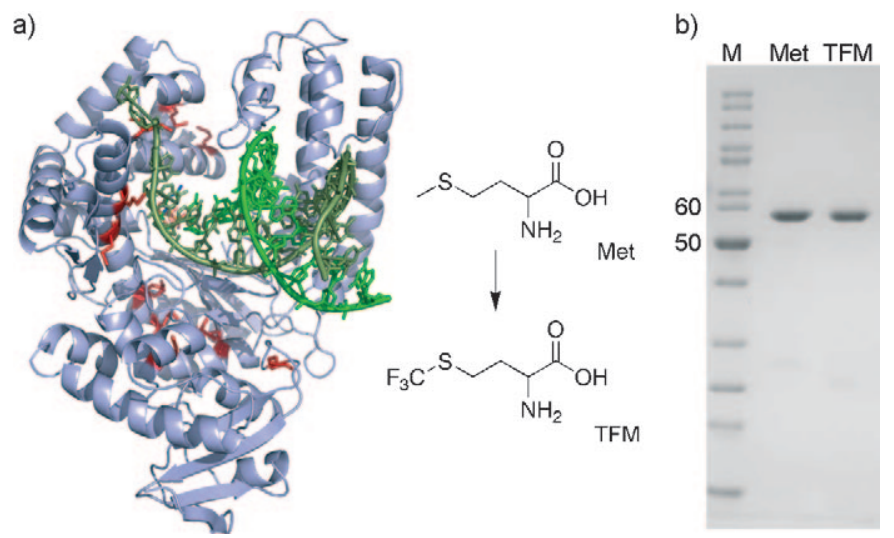

Scheme 1. Multi TFM labeled KlenTaq DNA polymerase. a) Methionine residues (Met) in KlenTaq DNA polymerase (PDB code: $3 \mathrm{KTQ}$ ) are highlighted in red. b) SDS PAGE gel of purified KlenTaq wt (Met) and KlenTaq TFM (TFM). M: Marker [kDa].

Taq was highly active and exhibited a similar selectivity as the wild type (wt) enzyme. Moreover, the introduction of the NMR active nucleus ${ }^{19} \mathrm{~F}$ offers the possibility to study DNA polymerase dynamics by ${ }^{19} \mathrm{~F}$ NMR spectroscopy. Despite its large size of $63 \mathrm{kDa}$, at least nine individual ${ }^{19} \mathrm{~F}$ resonances are observed, which allow us to distinguish different states of the DNA polymerase on the way to incorporating a canonical or a noncanonical nucleotide. To our knowledge, this is by far the largest enzymatically active protein with Met globally replaced by TFM.

First, we incorporated TFM into the KlenTaq DNA polymerase using the Met auxotrophic E. coli strain B834 (DE3) in a defined minimal medium. Thus, after depletion of Met, we incubated the cells for several hours in stationary phase, washed and resuspended them, added D,L TFM, and induced protein expression. We used the racemic mixture of TFM, as it is assumed that E. coli incorporates only the $\mathrm{L}$ form into proteins. KlenTaq wt was expressed using the same E. coli strain in standard LB medium. Both enzymes were purified to homogeneity (Scheme $1 \mathrm{~b}$ ). The substitution level of KlenTaq TFM was analyzed by electrospray ionization mass spectrometry (ESI MS; Figure 1 and the Supporting Information). Comparison of the molecular weights of KlenTaq TFM and the wild type shows that on average approximately $82 \%$ of the 14 Met amino acids (13 from KlenTaq gene, 1 initial Met) were replaced by TFM. By 


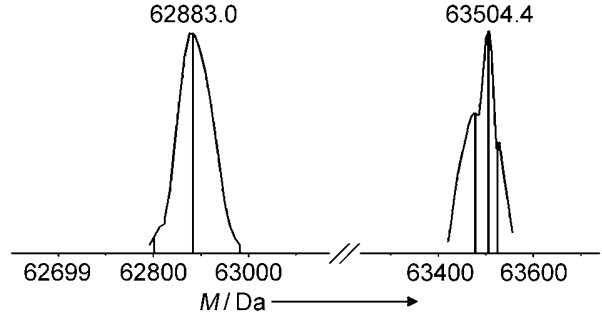

Figure 1. Deconvoluted ESI mass spectra profiles of KlenTaq wild type (62883.0 Da, calcd: $62830 \mathrm{Da}$ ) and KlenTaq TFM (63504.4 Da). M: molecular mass. TFM: trifluoromethionine. See also the Supporting Information.

performing a tryptic digest and analyzing the fragments with a combination of liquid chromatography (LC) and ESI MS, we were able to identify seven of the 14 Met positions (see the Supporting Information). At position M673 we detected only TFM, while at the other six positions we found the Met bearing peptide fragment as well as the TFM modified one. This result indicates that every Met is fluorinated to some extent.

Next, we investigated whether the highly modified KlenTaq TFM is still enzymatically active. We carried out primer extension reactions at temperatures between 37 and $70{ }^{\circ} \mathrm{C}$ (Figure $2 \mathrm{a}$ ). Both enzymes, KlenTaq TFM and wild

a)

Primer (23 nt): $\quad 5^{\prime}---G G$

Template (35 $\mathrm{nt}): 3^{\prime---C C A ~ A C T ~ A A A-5 ~}$
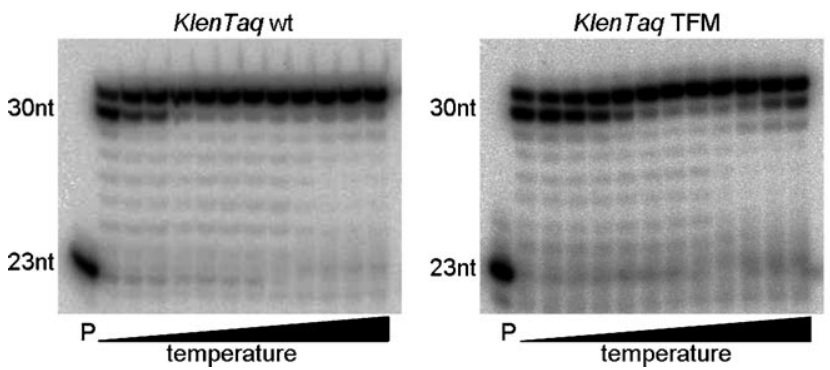

Figure 2. Enzymatic activity of KlenTaq TFM. a) Primer extension reactions catalyzed by KlenTaq wt and KlenTaq TFM. Partial primer template sequences employed are depicted on top. P: primer only; temperature: primer extension at 37.0, 37.8, 40.1, 43.7, 47.6, 51.5, 55.4, $59.4,63.3,66.9,69.2$, and $70.0^{\circ} \mathrm{C}$. b) PCR amplification of a 1655 bp fragment by KlenTaq wt (Met) and KlenTaq TFM (TFM). PCR program: initial $30 \mathrm{~s}$ at $95^{\circ} \mathrm{C}$ and 35 cycles with $20 \mathrm{~s}$ at $95^{\circ} \mathrm{C}, 60 \mathrm{~s}$ at $65^{\circ} \mathrm{C}$, and $120 \mathrm{~s}$ at $72^{\circ} \mathrm{C}$. M: Marker [bp], wt: wild type.

type, were able to extend the primer strand to full length in every case (30 nucleotides (nt)). The incorporation of an additional nucleotide in a nontemplated manner led to $31 \mathrm{nt}$ long products. This result has been observed before using $3^{\prime} \rightarrow$ $5^{\prime}$ exonuclease deficient DNA polymerases. ${ }^{[10]}$ By performing primer extension reactions at $72{ }^{\circ} \mathrm{C}$, we determined the specific activities of the DNA polymerases and observed that KlenTaq TFM retains more than one third of the activity of the wt enzyme (see the Supporting Information). Hence, the multifluorinated KlenTaq TFM is still a highly active DNA polymerase, even at temperatures above $70^{\circ} \mathrm{C}$.
To investigate whether the high Met $\rightarrow$ TFM substitution level affects the stability of the protein, we studied the thermostability of KlenTaq TFM in comparison to the wt. The enzymes were incubated for different periods of time at $95^{\circ} \mathrm{C}$. Subsequently, their activity was examined in primer extension reactions. We observed that KlenTaq TFM lost stability and exhibited less than $50 \%$ primer extension activity after incubation at $95^{\circ} \mathrm{C}$ for more than $30 \mathrm{~min}$, whereas even after five hours at $95^{\circ} \mathrm{C}$ the wt showed an activity of $90 \%$ (see the Supporting Information). Nevertheless, it turned out that KlenTaq TFM is still PCR active and is still able to amplify even rather long PCR products (Figure $2 \mathrm{~b}$ ). Furthermore, in real time PCR experiments the activity of KlenTaq TFM was similar to the wt activity (see the Supporting Information).

To quantify the selectivity of KlenTaq TFM, we deter mined the error spectra of the enzyme employing a reported PCR based assay (see the Supporting Information). ${ }^{[1]}$ The error rate of $3.2 \times 10^{-5}$ is quite similar to that found for wild type KlenTaq $\left(8.8 \times 10^{-5}\right) \cdot{ }^{[12]}$ In summary, our studies show that the global substitution of Met by TFM had most impact on the stability of the enzyme. Although the $\mathrm{CH}_{3} \rightarrow \mathrm{CF}_{3}$ substitution definitely increases the steric bulk of Met, ${ }^{[4,7]}$ and despite the fact that the Met residues are mainly located in the core of KlenTaq DNA polymerase, activity and fidelity are almost unchanged. Thus, the use of a highly stable protein enables the incorporation of trifluorinated amino acids with high substitution levels even in the core of b)

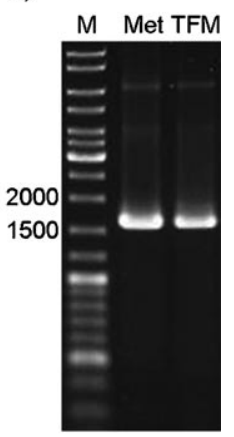
an enzyme without disturbing enzymatic activity significantly.

Several structural and functional stud ies of KlenTaq indicate that the enzyme undergoes significant conformational changes upon DNA and nucleotide bind ing. ${ }^{[13]}$ The introduction of ${ }^{19} \mathrm{~F}$ by TFM substitution of methionine allows the dynamics of the enzyme to be studied during substrate recognition and DNA synthesis. Thus, we investigated the enzyme by ${ }^{19}$ F NMR spectroscopy. Free KlenTaq TFM without any substrates dis plays resonances grouped around the ${ }^{19} \mathrm{~F}$ chemical shift of the TFM amino acid at $\delta=41 \mathrm{ppm}$. From the expected 14 reso nances corresponding to the 14 sequence positions of TFM, at least nine distinct resonances $($ at $\delta=38.31,39.20,39.42$, $39.68, \quad 40.12, \quad 40.52, \quad 40.95, \quad 41.03$, and $41.39 \mathrm{ppm}$ ) can be distinguished (Figure $3 \mathrm{~A}$ ). These resonances show significant differential line broadening ranging from $12 \mathrm{~Hz}$ of the sharpest signal at $\delta=40.95 \mathrm{ppm}$ to approximately $80 \mathrm{~Hz}$ of the broad signals at $\delta=38.31$ and $41.39 \mathrm{ppm}$.

These differences are most likely caused by different relaxation times of TFM residues at different positions in the 3D structure of KlenTaq TFM. It can be expected that $\mathrm{CF}_{3}$ groups located at surface exposed, highly flexible positions exhibit much smaller line widths than residues in the hydro phobic core of the protein, owing to shorter local rotational correlation times and the lower number of protons as sources 

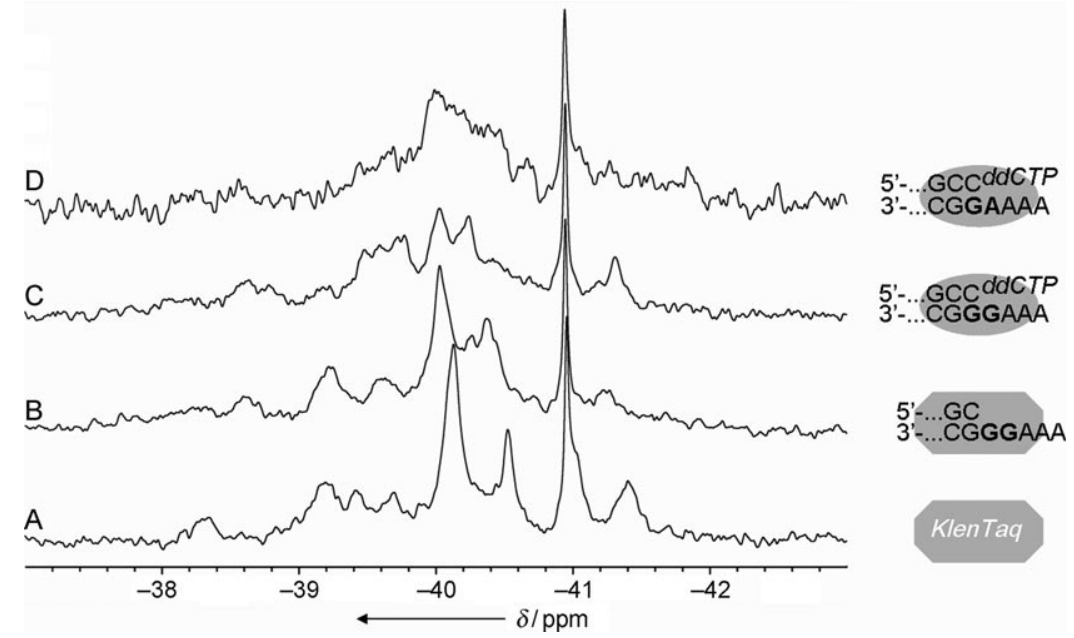

Figure 3. ${ }^{19} \mathrm{~F}$ NMR spectra of KlenTaq TFM A) without nucleic acid, B) with 2 equiv DNA substrate (GG), C) with 2 equiv DNA substrate (GG) and after addition of 10 equiv ddCTP, and D) with 2 equiv of a different DNA substrate (GA) and after addition of over 100 equiv ddCTP. Enzyme concentrations for (A C) were $42 \mu \mathrm{M}$ and $18 \mu \mathrm{M}$ for (D); intensity was adjusted to (A C)

of fast dipolar relaxation. Heterogeneity caused by the incomplete substitution of Met by TFM may broaden the peaks as well. We therefore conclude that the sharp resonance at $\delta=40.95 \mathrm{ppm}$, observed in all spectra irrespective of the presence of substrates, originates from the initial TFM residue located in the presumably highly flexible $\mathrm{N}$ terminus. All other resonances are more or less strongly affected by adding substrates. Upon addition of a DNA primer/template com plex leading to the formation of a binary protein DNA complex, several sharp resonances (full width at half max imum less than $40 \mathrm{~Hz}$ ) show up at new chemical shifts $(\delta=$ $40.03,40.37$, and $41.24 \mathrm{ppm})$, and the appearance of the other, broader signals also changes, for example, around $\delta=$

38.31 or $39.40 \mathrm{ppm}$ (Figure 3B). Subsequent addition of $2^{\prime}, 3^{\prime}$ dideoxycytidine $5^{\prime}$ triphosphate (ddCTP) should lead to phosphodiester formation with one ddCTP and formation of the ternary complex with a second ddCTP bound in the active site. ${ }^{[13]}$ Indeed, the spectrum of the ternary complex (Fig ure $3 \mathrm{C}$ ) is markedly different from those of both the free DNA polymerase and the binary complex, now with relatively sharp signals at $\delta=40.02,40.23$, and $41.30 \mathrm{ppm}$. Again, the broader resonances also change their appearance, for example, around $\delta=38.69$ or $39.59 \mathrm{ppm}$. Taken together, this evidence is indicative of another conformational change of KlenTaq TFM upon interaction with ddCTP.

Performing the same experiments with a DNA template that contains adenine (A) instead of guanine $(\mathrm{G})$ at the appropriate site in the template leads to the formation of a mismatched C A pair upon addition of ddCTP. We found that the ${ }^{19} \mathrm{~F}$ NMR spectrum differs from the match case. Almost all $\mathrm{CF}_{3}$ resonances of the mismatch complex (Figure 3D) suffer from severe line broadening. Apart from the signal at $\delta=$ $40.95 \mathrm{ppm}$ that we attribute to the $\mathrm{N}$ terminal TFM residue (see above), there are no resonances with sharp lines. Instead, a rather broad signal hump is present around $\delta=40.2 \mathrm{ppm}$. This finding suggests major changes of protein dynamics or the presence of structural heterogeneity in the mismatch case at the relatively high concen tration of ddCTP used herein to ensure saturation of the nucleoside triphosphate binding site. These structural changes might contribute to the selectivity of KlenTaq TFM by reducing the efficiency of noncanonical nucleotide incorporation. Thus, using ${ }^{19}$ F NMR spectroscopy we were able to clearly distinguish four different states of the DNA polymerase on the way to nucleotide incorpo ration.

In summary, we report the generation of a multifluorinated DNA polymerase that exhib its nearly the same enzymatic activity and fidelity as the parental enzyme. Owing to the high Met $\rightarrow$ TFM substitution level and the high signal intensity of the $\mathrm{CF}_{3}$ resonance even when incorporated into this comparatively large protein, the KlenTaq TFM serves as an optimal ${ }^{19} \mathrm{~F}$ NMR probe to elucidate altera tions of conformation and dynamics during enzyme catalysis. These findings open up new opportunities for further investigations, such as the directed evolution of DNA polymerases with new characteristics for future applications owing to the expanded amino acid repertoire ${ }^{[3,14]}$ or comparative studies of DNA polymerase dynamics by ${ }^{19} \mathrm{~F}$ NMR spectroscopy, to gain further insights into DNA synthesis fidelity and substrate spectra.

Keywords: DNA polymerases - NMR spectroscopy . non natural amino acids · protein engineering . trifluoromethionine

[1] A. Kornberg, T. A. Baker, DNA Replication, 2nd ed., Freeman, New York, 1991.

[2] J. Sambrook, D. W. Russell, Molecular Cloning: A Laboratory Manual, Cold Spring Harbor Laboratory Press, Cold Spring Harbor, 2001.

[3] a) D. Loakes, P. Holliger, Chem. Commun. 2009, 4619 4631; b) A. A. Henry, F. E. Romesberg, Curr. Opin. Biotechnol. 2005, 16, 370 377; c) S. Brakmann, Cell. Mol. Life Sci. 2005, 62, 2634 2646.

[4] a) N. Budisa, Engineering the Genetic Code, Wiley VCH, Weinheim, 2006; b) Y. Ryu, P. G. Schultz, Nat. Methods 2006, 3, 263265 ; c) L. Wang, P. G. Schultz, Angew. Chem. 2005, 117, 34 68; Angew. Chem. Int. Ed. 2005, 44, 34 66; d) A. J. Link, M. L. Mock, D. A. Tirrell, Curr. Opin. Biotechnol. 2003, 14, 603 609.

[5] C. Minks, S. Alefelder, L. Moroder, R. Huber, N. Budisa, Tetrahedron 2000, 56, 94319442.

[6] See, for example: a) J. C. Jackson, J. T. Hammill, R. A. Mehl, J. Am. Chem. Soc. 2007, 129, 1160 1166; b) J. T. Wong, Proc. Natl. Acad. Sci. USA 1983, 80, 6303 6306; c) E. A. Pratt, C. Ho, Biochemistry 1975, 14, 3035 3040; d) O. M. Rennert, H. S. Anker, Biochemistry 1963, 2, 471476.

[7] E. N. G. Marsh, Chem. Biol. 2000, 7, R153 R157. 
[8] a) N. Budisa, O. Pipitone, I. Siwanowicz, M. Rubini, P. P. Pal, T. A. Holak, M. L. Gelmi, Chem. Biodiversity 2004, 1, 1465 1475; b) H. Duewel, E. Daub, V. Robinson, J. F. Honek, Biochemistry 1997, 36, 34043416.

[9] W. M. Barnes, Gene 1992, 112, 2935.

[10] J. M. Clark, Nucleic Acids Res. 1988, 16, 96779686.

[11] P. H. Patel, H. Kawate, E. Adman, M. Ashbach, L. A. Loeb, J. Biol. Chem. 2001, 276, 50445051.

[12] C. Gloeckner, K. B. M. Sauter, A. Marx, Angew. Chem. 2007, 119, 3175 3178; Angew. Chem. Int. Ed. 2007, 46, 31153117.
[13] a) W. J. Allen, P. J. Rothwell, G. Waksman, Protein Sci. 2008, 17, 401 408; b) P. J. Rothwell, G. Waksman, J. Biol. Chem. 2007, 282, 28884 28892; c) P. J. Rothwell, V. Mitaksov, G. Waksman, Mol. Cell 2005, 19, 345355.

[14] a) C. C. Liu, A. V. Mack, M. L. Tsao, J. H. Mills, H. S. Lee, H. Choe, M. Farzan, P. G. Schultz, V. V. Smider, Proc. Natl. Acad. Sci. USA 2008, 105, 17688 17693; b) T. H. Yoo, A. J. Link, D. A. Tirrell, Proc. Natl. Acad. Sci. USA 2007, 104, 13887 13890; c) J. K. Montclare, D. A. Tirrell, Angew. Chem. 2006, 118, 4630 4633; Angew. Chem. Int. Ed. 2006, 45, 45184521. 